\title{
Prediction of Progression of Left Ventricular Hypertro- phy in Mild Hypertension: 5-Year Observations without Pharmacological Intervention
}

\author{
Hirofumi Tomiyama, Nobutaka Doba*, Toshio Kushiro, Makoto Yamashita, \\ Katsuo Kanmatsuse, Shigeaki Hinohara**, and Nagao Kajiwara
}

\begin{abstract}
We investigated the clinical implications of blood pressure changes after 20 min of rest and during exercise in particular for predicting the progression of left ventricular hypertrophy (LVH) in unmedicated male subjects with borderline and mild hypertension. We studied 155 subjects who were enrolled in a hypertension management program that included routine annual physical check-ups and cardiovascular follow-up studies, including echocardiography and treadmill exercise stress testing, every 3 years. Sixtynine subjects were followed for 5 years without pharmacological intervention. Left ventricular mass index (LVMI) was correlated with systolic pressure at peak exercise (max Ps) and 20-min resting systolic pressure in 155 subjects. Stepwise multiple linear regression analysis showed that the initial LVMI and max Ps had significantly higher percent explained variances in relation to the LVMI increase over the 5year observation period compared with other variables. Hyper-responders, subjects with an initial max Ps above $200 \mathrm{mmHg}$, showed a greater increase in LVMI than subjects with an initial max Ps below 200 $\mathrm{mmHg}$, although there was a small but significant increase in LVMI, without any increase in the blood pressure variables, even in those showing a normal response. In conclusion, max Ps appears to be a simple and reliable predictor of LVH progression. (Hypertens Res 1992; 15: 155-161)
\end{abstract}

Key Words: left ventricular hypertrophy, mild hypertension, systolic pressure at peak exercise

Because the final goal of management of essential hypertension is to improve and/or prevent cardiovascular morbidity and mortality (1), timely and appropriate intervention is essential. Most physicians who treated hypertensive patients are dissatisfied with the currently recommended WHO/ISH guidelines on the definition and management strategies of mild hypertension based on casually determined blood pressure levels (2-5).

Epidemiologic and clinical studies have shown that left ventricular hypertrophy (LVH) has an important role in the morbidity and mortality associated with hypertension $(6-8)$. We therefore investigated the predictive value of various clinical variables for the progression of LVH in the early stage of essential hypertension in unmedicated male subjects.

\section{Methods}

Subjects

Male subjects who were found to satisfy the following criteria during an annual physical check-up at the Life Planning Center (LPC, Tokyo, Japan) were enrolled in a continuous hypertension management program: 1) initial diagnosis between the ages of 30 to 49 years; 2) diastolic blood pressure between 90 to $105 \mathrm{mmHg}$ on three consecutive measurements; 3) no pharmacological intervention during the previous three years; and 4) no regular physical exercise. Patients with secondary hypertension or other coexisting serious medical problems requiring specific treatment were excluded. Informed consent was obtained from all subjects. The present study involved subjects who were enrolled in the program after March 1981 and whose most recent follow-up cardiovascular studies were completed by the end of December 1990.

\section{Methods \\ Blood Pressure Measurements}

Upon admission to the program, casual blood pressures were measured in the office with the patients seated after at least 10 min of rest using the conventional cuff method with a mercury manometer. Systolic and diastolic pressures were determined at the 1 st and 5th phases, respectively, of Korotkoff's sounds. Twenty-min resting blood pressure was

From the Department of Cardiology, Nihon University School of Medicine, Surugadai Hospital, Tokyo, *Division of Cardiology, the Third Department of Internal Medicine, Teikyo University School of Medicine, Ichihara, **The Life Planning Center, Tokyo.

Address for Reprints: Nobutaka Doba, M.D., Division of Cardiology, The Third Department of Internal Medicine, Teikyo University School of Medicine, 3426-3, Anegasaki, Ichihara, Chiba 299-01, Japan.

Received June 29, 1992; accepted in revised form December 24, 1992. 
measured at 2-min intervals over a 20 -min period with subjects in the semisupine position using a fully automated unit (Dynamap 950, Tampa, USA). We determined the mean of 5 measurements taken over the last $10 \mathrm{~min}$. Blood pressures during exercise were obtained by the ordinary K-sound method using a mercury manometer. Blood pressure at peak exercise was defined as the pressure measured at the peak of the symptom-limited maximal treadmill exercise stress test according to the conventional Bruce protocol (9).

Echocardiographic Measurements

M-mode echocardiograms, guided by two-dimensional echocardiography, were obtained using a Sonolayer (SSH-1, Toshiba Co. Tokyo, Japan) with a $2.5-\mathrm{MHz}$ transducer. Data were printed on a split chart-recorder with a recording speed of $50 \mathrm{~mm} / \mathrm{s}$. Interventricular septal thickness (IVS), left ventricular internal diameter (LVD), and posterior wall thickness (PWT) were measured at. end-diastole according to the recommendations of The American Society of Echocardiography and the Penn Convention $(10,11)$. Each dimension was defined as the mean of two measurements, obtained from an Mmode recording, performed by different investigators.

Left ventricular mass (LVM), cardiac output (CO), ejection fraction (EF), and mean velocity of the left ventricular circumference $(\mathrm{mVcf})$ were calculated by the following formulae: $\mathrm{LVM}=1.04 \times$

$\left\{(\mathrm{LVDd}+\mathrm{PWTd}+\mathrm{IVSTd})^{3}-\mathrm{LVDd}^{3}\right\}-13.6, \mathrm{CO}$ $=(\mathrm{EDV}-\mathrm{ESV}) \times \mathrm{HR} . ; \mathrm{EF}=\left(1-\mathrm{LVDd}^{3} / \mathrm{LVDs}^{3}\right) \times$ $100 ;$ and $\mathrm{mVcf}=\{(\mathrm{LVDd}-\mathrm{LVDs}) / \mathrm{LVDd}\} \times \mathrm{ET}$. Abbreviations: LVDd (end-diastolic left ventricular diameter); LVDs (end-systolic left ventricular diameter); PWTd (end-diastolic posterior wall thickness); IVSTd (end-diastolic interventricular septal thickness); EDV (end-diastolic left ventricular volume); ESV (end-systolic left ventricular volume), and ET (ejection time). Both EDV and ESV were derived by Teichholtz's method (12). The left ventricular mass and cardiac indices (LVMI and CI) were calculated by dividing LVM and $\mathrm{CO}$ by the body surface area (BSA).

Program Follow-Up

Following routine health education, subjects entered the follow-up program, which involved annual physical check-ups and a cardiovascular evaluation every 3 years. If a subject's casual diastolic pressure exceeded $105 \mathrm{mmHg}$, he was asked to withdraw from the program, and pharmacological intervention was recommended.

Statistical Analysis

Data, arranged for the statistical package STAX, were expressed as the mean \pm 1 SD. Linear regression analysis was used to evaluate the correlation between LVMI and blood pressure variables. The relation of LVMI to initial blood pressure variables at the end of the follow-up period and the change in LVMI during the observation period (delta LVMI) were evaluated using stepwise multiple linear regression analysis. Results are expressed as a percentage of the explained variance $\left(R^{2}\right)$. We selected the following independent variables for analysis: LVMI; casual, rest and maximal blood pressures; age; body mass index; and duration of follow-up period. A probability value of less than 0.05 was considered statistically significant.

\section{Results}

General Observations

Suitable echocardiograms allowing for reliable measurement were obtained in 155 of 243 eligible subjects. Data from these 155 subjects (mean age $43 \pm 8$ years) were analyzed. By the end of 1990,69 of 155 subjects had been followed for over 3 years without pharmacological intervention. The median duration of follow-up was $4.9 \pm 1.8$ years. Of the remaining 86 subjects, 6 were recommended for phar-

Table 1. Demographics, Blood Pressure and Echocardiographic Variables in 155 Males

\begin{tabular}{lc} 
Age & $43 \pm 8$ \\
Body weight (kg) & $66 \pm 5$ \\
Casual Ps (mmHg) & $138 \pm 13$ \\
Casual Pd (mmHg) & $91 \pm 8$ \\
20 min resting Ps (mmHg) & $130 \pm 13$ \\
20 min resting Pd (mmHg) & $82 \pm 10$ \\
Maximal Ps (mmHg) & $199 \pm 23$ \\
Maximal Pd (mmHg) & $94 \pm 14$ \\
Maximal HR (bpm) & $174 \pm 14$ \\
Treadmill time (sec) & $709 \pm 118$ \\
LVMI (g/m $\left.{ }^{2}\right)$ & $105.9 \pm 29.6$ \\
Cardiac index (l/m $\left.{ }^{2}\right)$ & $2.6 \pm 0.7$ \\
Ejection fraction (\%) & $64.6 \pm 8.6$ \\
mVcf (circ/sec) & $1.1 \pm 0.2$ \\
\hline
\end{tabular}

\footnotetext{
Abbreviations: casual Ps (casual systolic pressure); casual Pd (casual diastolic pressure); 20-min resting Ps (20-min resting systolic pressure); 20 min resting Pd (20-min resting diastolic pressure); maximal Ps (systolic pressure at peak exercise); maximal Pd (diastolic pressure at peak exercise); maximal heart rate (heart rate at peak exercise); LVMI (left ventricular mass index); $\mathrm{mVcf}$ (mean velocity of circumferential shortening).
} 
macological intervention, 8 did not have a suitable echocardiogram at the second examination, 28 had been followed for less than 3 years, and 44 did not return for a second examination.

Table 1 shows the demographics, blood pressure and echocardiographic variables obtained in the 155 subjects at the initiation of the program. Anthropometric measurements and physical fitness levels showed that they were representative of typical middle-aged Japanese men with mild or borderline hypertension (13).

At the beginning of the observation period, 20min resting blood pressure and blood pressure at peak exercise were more strongly correlated with LVMI than was casual blood pressure (Fig.1). Blood pressure at peak exercise showed the best correlation with LVMI $(r=0.57, p<0.001)$.

\section{Progression of $L V H$}

After a follow-up period of 4.9 years, there was a marked decrease in physical fitness and no change in body weight, probably because of poor compliance with recommended non-pharmacological treatment regimens (Table 2). Although there were no changes in any blood pressure variables, LVMI showed a significant increase of $+16.7 \%$.
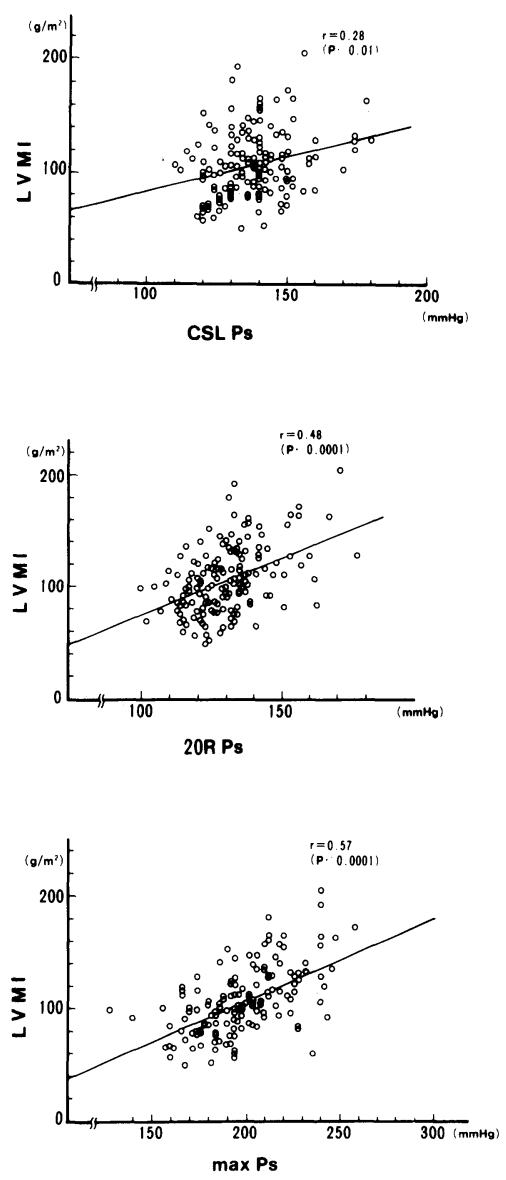

\section{Prediction of LVMI}

Figure 2 shows the percent explained variance of each variable for the prediction of the final LVMI and delta LVMI. When the initial LVMI was included in independent variables, Table 3 shows partial correlation coefficients and partial regression coefficients in that analysis. The initial LVMI had the greatest explained variance of $77.5 \%$ variability and other variables such as max Ps, the duration of follow-up, and age at the initiation of the observation only revealed $2.4 \%, 2.3 \%$, and $0.9 \%$ levels of explained variance, respectively (Fig. 2). Table 4 shows partial correlation coefficients and partial regression coefficients when the initial LVMI was excluded from analysis. The initial max Ps emerged to be the most important predictor with a $30.5 \%$ explained variance level (Fig. 2). The initial max Ps was also the strongest predictor of delta LVMI when initial LVMI was excluded from analysis, with a $14.1 \%$ explained variance (Fig. 2).

\section{Association between the Initial Max Ps and LVMI} Increase

Preliminary data suggested that a max Ps of 200 $\mathrm{mmHg}$ was a useful predictor of progression in LVH (14). Therefore, we divided subjects into two
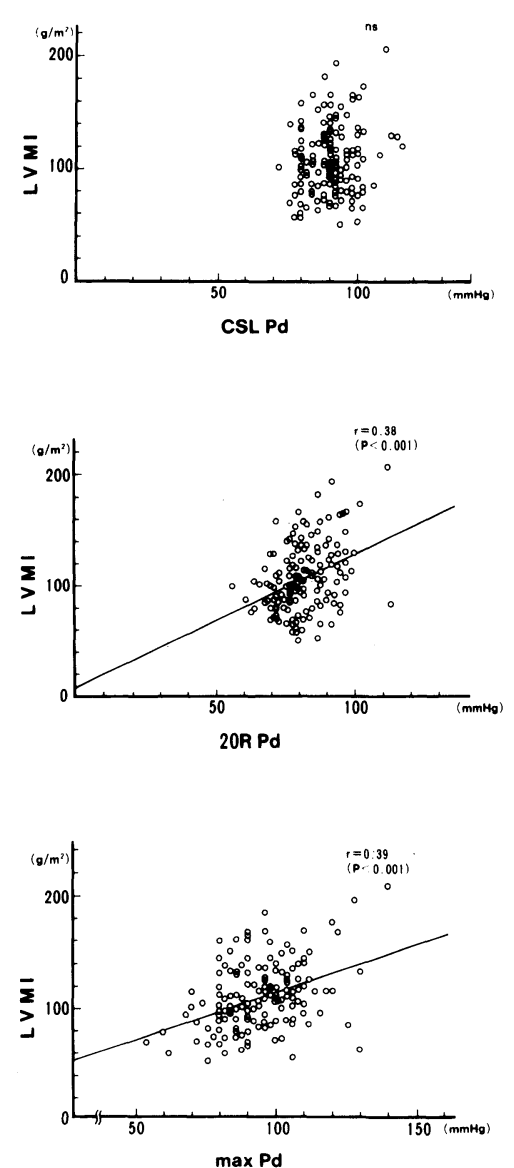

Fig. 1. Correlations of LVMI with blood pressure variables in 155 subjects at the beginning of the observation period. 
Table 2. Body Weight, Blood Pressure and Echocardiographic Variables in 69 Untreated Males Followed for 5 Years

\begin{tabular}{|c|c|c|c|}
\hline & Initial & Follow-up & $p$ value \\
\hline Body weight (kg) & $65 \pm 7$ & $65 \pm 8$ & ns \\
\hline Casual Ps (mmHg) & $141 \pm 12$ & $139 \pm 15$ & ns \\
\hline Casual Pd (mmHg) & $92 \pm 8$ & $92 \pm 10$ & ns \\
\hline 20-min resting Ps $(\mathrm{mmHg})$ & $130 \pm 10$ & $131 \pm 13$ & ns \\
\hline $20-$ min resting $\mathrm{Pd}(\mathrm{mmHg})$ & $82 \pm 8$ & $81 \pm 9$ & ns \\
\hline Maximal Ps (mmHg) & $199 \pm 22$ & $200 \pm 24$ & ns \\
\hline Maximal Pd (mmHg) & $94 \pm 14$ & $95 \pm 13$ & ns \\
\hline Maximal HR (bpm) & $175 \pm 18$ & $173 \pm 16$ & ns \\
\hline Treadmill time (sec) & $715 \pm 117$ & $662 \pm 105$ & $p<0.05$ \\
\hline $\operatorname{LVMI}\left(\mathrm{g} / \mathrm{m}^{2}\right)$ & $106.7 \pm 20.7$ & $124.5 \pm 28.8$ & $p<0.01$ \\
\hline Cardiac index $\left(1 / \mathrm{m}^{2}\right)$ & $2.6 \pm 0.8$ & $2.6 \pm 0.6$ & ns \\
\hline Ejection fraction (\%) & $66 \pm 7$ & $67 \pm 9$ & ns \\
\hline $\mathrm{mVcf}(\mathrm{circ} / \mathrm{sec})$ & $1.1 \pm 0.2$ & $1.1 \pm 0.2$ & ns \\
\hline
\end{tabular}

Abbreviations: casual Ps (casual systolic pressure); casual Pd (casual diastolic pressure); 20-min resting Ps (20-min resting systolic pressure); 20-min resting Pd (20-min resting diastolic pressure); maximal Ps (systolic pressure at peak exercise); maximal Pd (diastolic pressure at peak exercise); maximal heart rate (heart rate at peak exercise); LVMI (left ventricular mass index); $\mathrm{mVcf}$ (mean velocity of circumferential shortening).

Table 3. Predictive Value of Independent Variables Including Initial LVMI for Final LVMI

\begin{tabular}{lccc}
\hline & $\begin{array}{c}\text { partial correlation } \\
\text { coefficient }\end{array}$ & $\begin{array}{c}\text { partial regression } \\
\text { coefficient }\end{array}$ & $p$ value \\
\hline LVMI & 0.836 & 1.073 & $p<0.01$ \\
Casual Ps & -0.228 & $\mathrm{~ns}$ & $\mathrm{~ns}$ \\
Casual Pd & -0.250 & $\mathrm{~ns}$ & $\mathrm{~ns}$ \\
20-min resting Ps & 0.269 & $p<0.01$ \\
20-min resting Pd & -0.247 & $\mathrm{~ns}$ \\
Maximal Ps & 0.404 & 0.207 & $p<0.05$ \\
Maximal Pd & 0.107 & & $\mathrm{~ns}$ \\
Age & 0.184 & 0.416 & \\
Body mass index & -0.028 & & \\
Duration & 0.262 & 0.170 & \\
\hline
\end{tabular}

Abbreviations: casual Ps (casual systolic pressure); casual Pd (casual diastolic pressure); 20-min resting Ps (20-min resting systolic pressure); 20-min resting Pd (20-min resting diastolic pressure); maximal Ps (systolic pressure at peak exercise); maximal Pd (diastolic pressure at peak exercise); maximal heart rate (heart rate at peak exercise); LVMI (left ventricular mass index); $\mathrm{mVcf}$ (mean velocity of circumferential shortening).

subgroups based on their initial max Ps; subjects with a max $\mathrm{Ps} \geq 200 \mathrm{mmHg}$ were classified as hyperresponders and subjects with a $\max P s<200 \mathrm{mmHg}$ were classified as normal responders. Initial casual systolic blood pressure was not significantly different between the two subgroups. This variable showed no significant intragroup changes during the observation period, but the casual systolic blood pressure was significantly higher in hyper-responders than in normal responders after the 5-year observation period (Fig. 3). Initial LVMI was significantly greater in hyper-responders compared with normal responders, and hyper-responders showed a significantly greater increase in LVMI after 5 years than did normal responders.

\section{Discussion}

Clinical trials have demonstrated the effectiveness of treating essential mild hypertension $(15,16)$. Because of the high cost of antihypertensive treatment to all patients with providing mild hypertension, and because of the relatively low incidence of cardiovascular events in this group, treatment is not universally provided to such patients (17). Casual blood pressure is not considered to be a reliable measure of blood pressure or a valid indication of target organ damage, especially of LVH (18). Therefore, better measures are needed to evaluate the cardiovascular status of patients with mild hypertension. 
Table 4. Predictive Value of Variables Excluding Initial LVMI

\begin{tabular}{lccl}
\hline & $\begin{array}{c}\text { partial correlation } \\
\text { coefficient }\end{array}$ & $\begin{array}{c}\text { partial regression } \\
\text { coefficient }\end{array}$ & $p$ value \\
\hline Casual Ps & -0.017 & & $\mathrm{~ns}$ \\
Casual Pd & -0.231 & $\mathrm{~ns}$ & $p<0.01$ \\
20-min resting Ps & 0.358 & 0.264 & $\mathrm{~ns}$ \\
20-min resting Pd & -0.185 & & $p<0.01$ \\
Maximal Ps & 0.385 & 0.367 & $\mathrm{~ns}$ \\
Maximal Pd & -0.003 & & $p<0.01$ \\
Age & 0.298 & 0.235 & $\mathrm{~ns}$ \\
Body mass index & -0.001 & & $p<0.01$ \\
Duration & 0.325 & 0.235 & \\
\hline
\end{tabular}

Abbreviations: casual Ps (casual systolic pressure); casual Pd (casual diastolic pressure); 20-min resting Ps (20-min resting systolic pressure); 20-min resting Pd (20-min resting diastolic pressure); maximal Ps (systolic pressure at peak exercise); maximal Pd (diastolic pressure at peak exercise); maximal heart rate (heart rate at peak exercise); LVMI (left ventricular mass index); $\mathrm{mVcf}$ (mean velocity of circumferential shortening).

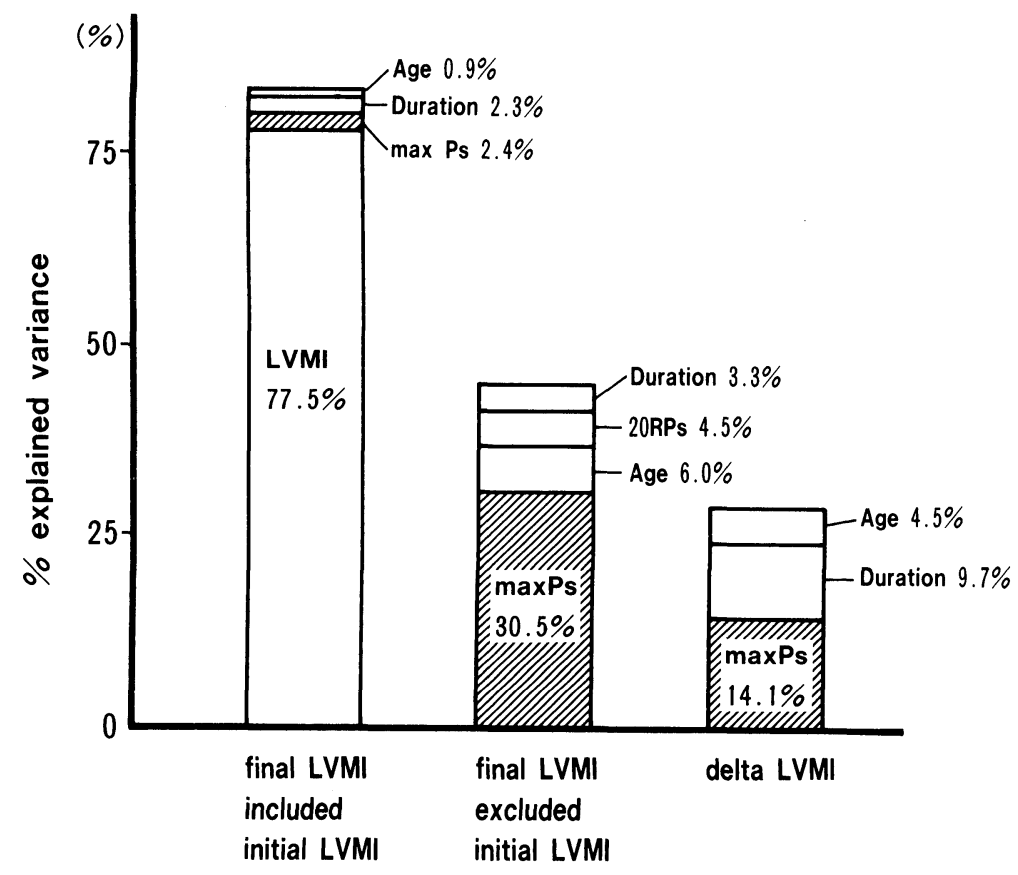

Fig. 2. Results of stepwise multiple linear regression analysis of variables for prediction of final LVMI after a 5-year follow-up and increase in LVMI during follow-up. The vertical line represents the percent explained variances of independent variables. The horizontal line represents dependent variables. Abbreviations: LVMI (left ventricular mass index); 20RPs (20-min resting systolic pressure); max Ps (systolic pressure at peak exercise); Duration (the duration of follow-up); final LVMI included initial LVMI (The final LVMI is the dependent variable and the initial LVMI is included in independent variables.); final LVMI excluded initial LVMI (The final LVMI is the dependent variable and the initial LVMI is excluded from independent variables.); delta LVMI (Change in LVMI during the observation period is the dependent variable).

Our results showed that max Ps was a useful indicator of the current state of $\mathrm{LVH}$, and that both the initial LVMI and max Ps were independent predictors of the progression of LVH. Subsequent analysis showed that a blood pressure level at peak exercise above $200 \mathrm{mmHg}$ was the most reliable predictor of LVH progression. Although systolic blood pressure at exercise has been identified as a predictor of LVH in cross-sectional studies, the present study is the first longitudinal study to our knowledge, to investigate the clinical significance of max Ps for prediction of LVH progression.

Casual blood pressure has not been found to be a reliable measure of LVH (18). The 20-min resting 

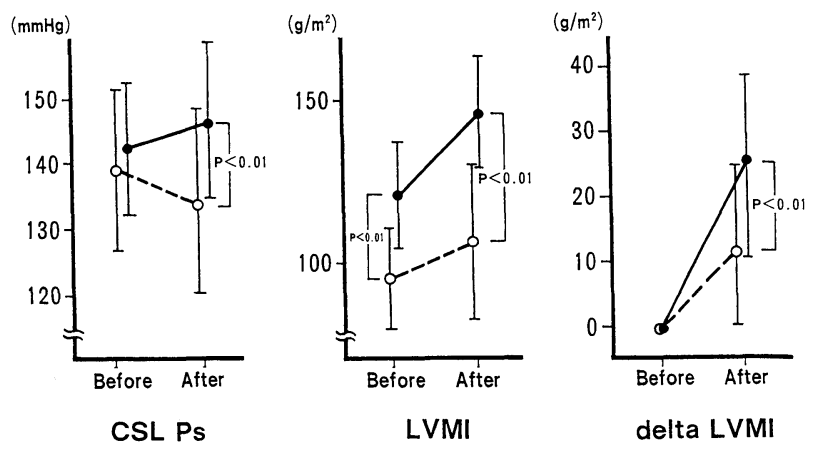

Fig. 3. Changes in blood pressure and LVMI variables in subjects classified according to systolic pressure at peak exercise. Open circles represent the normal responder group and closed circles represent the hyper-responder group. Abbreviations: CSLPS (casual systolic pressure); LVMI (left ventricular mass index); delta LVMI (change of LVMI during the observation period).

blood pressure measure used in the present study represented the average of 5 consecutive measurements obtained at 2-min intervals over the final 10 min using a fully automated device. This $20-\mathrm{min}$ resting blood pressure measurement has been found to be significantly correlated with the 24 -h average and midnight sleep blood pressure levels (19). Although the average 24-h systolic pressure has been reported to be well correlated with LVH (20, 21 ), the 20-min resting systolic blood pressure is relatively easy to obtain and our results showed that it was significantly correlated with LVMI. This measure would seem to be useful for estimating the presence of LVH, but its low percent of explained variance suggests that it is not a good predictor of LVH progression.

Fagard et al. reported that blood pressure measurement obtained during exercise did not offer any additional precision in predicting cardiovascular morbidity and mortality over the use of resting blood pressure and age (22). However, the age range in their patient population was extremely wide, and more than half of their subjects had moderate hypertension. Cardiovascular morbidity in patients with mild hypertension between the ages of 40 and 60 years is lower than in those over 60 years of age (23). Therefore, the clinical significance of the exercise blood pressures in the Fagard et al. study is questionable because of the relatively older age of their patients and, consequently, their relatively advanced hypertension. Max Ps may be a clinically useful prognostic factor only in the relatively early stage of hypertension.

Koren et al. reported that LVMI and left ventricular geometry are more useful than casual blood pressure for predicting cardiovascular events (24). Echocardiography is simpler and more cost-effective than the exercise test. However, our results showed that max Ps was a predictor of final LVMI and progression of LVMI independently of initial
LVMI. Thus, max Ps may provide useful information in addition to echocardiographic study for estimating the progression of mild hypertension.

The present study raises the question of why an exaggerated blood pressure response during exercise is one of the best predictors of LVH progression. It has been suggested that both hemodynamic and neurohormonal factors contribute to the progression of LVH (25). Gottodinner et al. reported that LVH was present even in normotensives whose systolic blood pressure at peak exercise exceeded 210 $\mathrm{mmHg}(26)$, suggesting that a hyperdynamic response to peak exercise is not a cause of $\mathrm{LVH}$, but a result (27). It may be that both hypertension and LVH are caused by some other as yet unspecified factors, and that they may progress simultaneously, with systemic effects. Further studies are needed to clarify these issues.

Many blood pressure variables showed no change during the 5-year observation period. However, when subjects were classified as hyper-responders or normal responders according to their initial max Ps, casual systolic pressures differed significantly after 5 years in association with a relatively large LVH progression in the hyper-responder group. Studies have shown that blood pressure is not always elevated in its course and sometimes improves without pharmacological intervention $(15,28)$. Our results also support these findings, and indicate that a max Ps above $200 \mathrm{mmHg}$ is a reliable measure for predicting the progression of mild hypertension. The present study demonstrated small but significant increases in LVMI without progressive elevation of blood pressure variables, providing further evidence that $\mathrm{LVH}$ may progress in the absence of increases in blood pressure.

In conclusion, max Ps emerged as a simple and useful factor for predicting the progression of $\mathrm{LVH}$. Further study is required to confirm its long-term prognostic significance.

\section{Acknowledgment}

The authors wish to express their deep appreciation to Mrs. Teruko Kitagawa at the Life Planning Center for her excellent technical assistance in echocardiography, and to Dr. Yoichi Kuwabara at the Third Department of Internal Medicine, Chiba University School of Medicine for his critical review of the manuscript.

\section{References}

1. Kaplan NM: Treatment of hypertension: Rationale and Goal, in Kaplan NM (ed.): Clinical Hypertension. Baltimore, Williams \& Wilkins, 1990, pp136162.

2. 1989 Guidelines for the management of mild hypertension: memorandum from a WHO/ISH meeting. $J$ Hypertens 1989; 7: 689-693.

3. Mancia G, Casadei R, Groppelli A, Parati G, Zancheti A: Effect of stress on diagnosis of hypertension. Hypertension 1991; 17 [Suppl III]: III-56-III-62.

4. Sokolow M, Werdegar S, Kain H, Hinman AT: Relationship between level of blood pressure measured 
casually and by portable recorders and severity of complications in essential hypertension. Circulation 1966; 34: 279-298.

5. Cutler JA, MacMahon SW, Furberg CD: Controlled clinical trials of drug treatment of hypertension. A review. Hypertension 1989; 13: I36-I44.

6. Messerli FH, Ventura HO, Elizardi DJ, Frohlich ED: Hypertension and sudden death: Increased ventricular ectopic activity in left ventricular hypertrophy. Am J Med 1984; 77: 18-22.

7. Marcus ML, Harrison DG, Chilian WN, et al: Alterations in the coronary circulation in hypertrophied ventricules. Circulation 1987; 75 (suppl I): I19-I25.

8. Casale PN, Devereoux RB, Milner M, et al: Value of echocardiographic measurement of left ventricular mass in predicting cardiovascular morbid events in hypertensive men. Ann Int Med 1986; 105: 173-178.

9. Bruce RA, Kusumi F, Hosner D: Maximal oxygen intake and nomographic assessment of functional aerobic impairment in cardiovascular disease. $\mathrm{Am}$ Heart J 1973; 85: 546-562.

10. Sahn DJ, DeMaria A, Kisslo J, Weyman A: Recommendations regerding quantification in M-mode echocardiography: results of a survey of echocardiographic measurements. Circulation 1978; 58: 10721083.

11. Devereoux RB, Reichek N: Echocardiographic determination of left ventricular mass in man: anatomic validation of the method. Circulation 1977; 55: 613618.

12. Teichholz LE, Kreulen T, Herman MV: Problems in echocardiographic volume determinations; Echocardiographic and angiographic correlations in the presence or abscence of asynergy. Am J Cardiol 1976; 37: 7-14.

13. Doba N, Fukui T, Yamashina A, et al: Functional evaluation of the oxygen transport system with multiphasic treadmill stress testing after Bruce's protcol: Racial difference in cardiovascular response to exer cise. Kokyu to Jyunkan 1980; 28: 507-512 (in Japanese).

14. Tomiyama H: Usefulness of symptom-limited exercise test in the evaluation of cardiac hypertrophy and arteriolar damage in mild essential hypertension. $J$ Nihon Univ Med Ass 1989; 48: 837-841 (in Japanese).

15. The Australian therapeutic trial in mild hypertension. Report by the Management Committee. Lancet 1980; 1: 1261-1267.

16. Medical Research Council Working Party. MRC trial of treatment of mild hypertension: principal results Br Med J Clin Res 1985; 291: 97-104.
17. Report of the British Hypertension Society Working Party: Br Med J 1989; 298: 694.

18. Devereoux RB, Pickering TG, Harshfield GA, et al: Left ventricular hypertrophy in patients with hypertension: Importance of blood pressure response to regularly reccurring stress. Circulation 1983; 68: 470482 .

19. Doba N, Kushiro T, Yamashita M, et al: Determination of the blood pressure level in mild hypertension. Significance of 20 minute resting blood pressure measurement comparing to 24 hour ambulatory blood pressure monitoring. Kokyu to Junkan 1989; 37: 903-908 (in Japanese).

20. Verdecchia P, Schilaci G, Boldrini F, Guerrieri M, Gatteschi C, Benemio G, Porcellati C: Risk stratification of left ventricular hypertrophy in systemic hypertension using noninvasive ambulatory blood pressure monitoring. Am J Cardiol 1990; 66: 583590.

21. Meyer-Sabellek WA, Schulte KL, Liederwald K, Gemmeren D, Gotzen R: Blood pressure profile and cardiac risk in hypertensive patients with left ventricular hypertrophy. J Hypertens 1990; 8 [Suppl 4]: S95-S98.

22. Fagard R, Staessen J, Thijs L, Amery A: Prognostic significance of exercise versus resting blood pressure in hypertensive men. Hypertension 1991; 17: 574-578.

23. Veterans Administration Cooperative Study Group on antihypertensive agents. Effects of treatment on morbidity in hypertension. III. Influence of age, diastolic blood pressure and prior cardiovascular disease: further analysis on side effects Circulation 1972; 45: 991-1004.

24. Koren MJ, Devereoux RB, Casale PN, Savage DD, Laragh JH: Relation of left ventricular mass and geometry to morbidity and mortality in uncomplicated essential hypertension. Ann Intern Med 1991; 114: 345-352.

25. Frohlich ED: The heart in hypertension. Hypertension 1991; 18 [Suppl III]: III-62-III68.

26. Gottdiener JS, Brown J, Zoltick J, Fletcher RD: Left ventricualr hypertrophy in men with normal blood pressure: Relation to exaggerated blood presure response to exercise. Ann Intern Med 1990; 112: 161166.

27. Lund-Johansen P: Hemodynamic alterations in early essential hypertension: Recent advances; in Gross F and Strasser T (eds): Mild hypertension: Resent advances. New York, Raven Press, 1983, pp237-246.

28. Stewart IMG: Lond-term observations on high bloodpressure presenting in fit young men. Lancet 1971; 1: 355-358. 